\title{
Development of a Molecularly Imprinted Electrochemical Sensor for Tert-Butylhydroquinone Recognition
}

\author{
Rongzhou Qin ${ }^{1}$, Qilin Wang ${ }^{1}$, Chaoqin Ren ${ }^{1}$, Xianzhi Dai ${ }^{1}$ and Haijun Han ${ }^{2, *}$ \\ ${ }^{1}$ Aba Normal University, Shuimo, Wenchuan, Aba Autonomous Prefecture of Tibetan and Qiang, \\ Sichuan, 623001, China \\ ${ }^{2}$ Sichuan University, No.24 South of the Yihuan Rd, Chengdu, Sichuan, 610065, China \\ *E-mail: hhj-zhongzhouzidao@zidao.co
}

doi: $10.20964 / 2017.10 .04$

Received: 30 May 2017 / Accepted: 24 July 2017 / Published: 12 September 2017

\begin{abstract}
In this work, a new molecularly imprinted voltammetric sensor was fabricated based on a glassy carbon electrode (GCE) modified with reduced graphene oxide (rGO)-based silver nanoparticles (AgNPs) for the detection of tert-butylhydroquinone (TBHQ). X-ray diffraction (XRD) and X-ray photoelectron spectroscopy (XPS) techniques were used for the characterization of the proposed surfaces. The linearity range of the proposed method was determined to be $0.05-1.5 \mathrm{nM}$, and the limit of detection (LOD) was $1.48 \times 10^{-11} \mathrm{M}$. The new voltammetric sensor exhibited remarkable recovery and selectivity for the detection of real specimens. The stability of the sensor was also investigated.
\end{abstract}

Keywords: Butylhydroquinone; Molecular imprinting; Graphene; Voltammetric sensor; Food samples

\section{FULL TEXT}

(C) 2017 The Authors. Published by ESG (www.electrochemsci.org). This article is an open access article distributed under the terms and conditions of the Creative Commons Attribution license (http://creativecommons.org/licenses/by/4.0/). 